Pacific Journal of Mathematic 


\section{THE BACK-AND-FORTH ISOMORPHISM CONSTRUCTION}

\section{DALe Myers}

Frequently in model theory and occasionally elsewhere, a back-and-forth construction is used to show that any two countable structures satisfying a given relation are isomorphic. Such a construction is used to show that any two countable dense linear orders without end points are isomorphic (Cantor), that any two countable reduced $p$-primary abelian groups with the same Ulm invariants are isomorphic (see Kaplansky, In finite Abelian Groups), and that any two countable elementarily equivalent saturated structures are isomorphic (Morley and Vaught). The back-and-forth arguments using these constructions can often be reduced to an application of the following result: If $R$ is a symmetric relation between countable structures such that $(1) \mathfrak{U} R \mathfrak{B}$ implies $\mathfrak{U}$ and $\mathfrak{B}$ satisfy the same atomic sentences and (2) $\mathfrak{A} R \mathfrak{B}$ and $a \in \mathfrak{A}$ implies there is a $b \in \mathfrak{B}$ such that $(\mathfrak{Y}, a) R(\mathfrak{B}, b)$, then $\mathfrak{A} R \mathfrak{B}$ implies $\mathfrak{A} \cong \mathfrak{B}$.

Loosely, the second condition requires that related structures have enough related expansions by constants. We prove a similar result in which the second condition requires, loosely, that related structures have enough similar decompositions into related components. The prototype of our result is a theorem of Vaught's on Boolean algebras mentioned in the last section. In order to suitably formalize "decomposition" we use category theory.

In a subsequent paper, our two theorems will be used to solve the problem of determining when two free locally-finite cylindric algebras are isomorphic. In particular, the answer to both questions in problem 2.8 of $[4, \mathrm{p} .463]$ is no. The answer to the first is yes if $|\Delta \xi|=\kappa$ and $\left|\Delta^{\prime} \xi\right|=\kappa$ are replaced by $|\Delta \xi| \geqq \kappa$ and $\left|\Delta^{\prime} \xi\right| \geqq \kappa$ respectively.

Constructors and decomposable relations. We shall use [7] as our notational reference. A directed category is a small category generated by a preorder for which every pair of elements has an upper bound. Let $\underline{C}$ be a category whose arrows are monics. Let $\underline{C}^{*}$ be the category whose objects are functors from directed categories to $\underline{C}$ and for which an arrow from an object $A: \alpha \rightarrow \underline{C}$ to an object $B: \bar{\beta} \rightarrow \underline{C}$ is an ordered pair $\langle f, \tau\rangle$ such that $f: \alpha \rightarrow \beta$ is a functor and $\tau: A \rightarrow$ $f \circ B$ is a natural transformation. The components of $\langle f, \tau\rangle: A \rightarrow B$ are those of $\tau$, i.e., the maps $\tau_{i}$ for $i$ in the domain of $A$. If $\langle f$, 
$\tau\rangle: A \rightarrow B$ and $\langle g, \sigma\rangle: A \rightarrow D$ and $i$ is in the domain of $A$, then $\tau_{i}$ and $\sigma_{i}$ are corresponding components. By identifying an object $a$ of $\underline{C}$ with the functor $1 \rightarrow \underline{C}$ whose unique object value is $a$, we can consider $\underline{C}$ to be a subcategory of $\underline{C}^{*}$. For an object $A$ of $\underline{C}^{*}$, an object $\bigcup A$ of $\underline{C}$ is a colimit of $A$ iff there is an arrow $A \rightarrow \bigcup A$ which is universal from $A$ to $\underline{C}$. Such an arrow is called a universal cone. An object $c$ in $\underline{C}$ is compact iff for every universal cone $A \rightarrow$

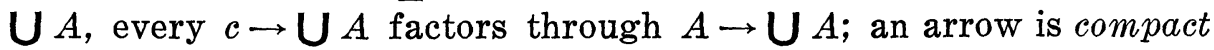
iff its domain is. (See the last section for examples.) A category is compactly generated iff for any two arrows $a \rightarrow_{1} b$ and $a \rightarrow_{2} b, a \rightarrow_{1} b \neq$ $a \rightarrow_{2} b$ implies $c \rightarrow a \rightarrow_{1} b \neq c \rightarrow a \rightarrow_{2} b$ for some compact arrow $c \rightarrow a$. An object in $\underline{C}$ is countable iff it is a colimit $\mathrm{U} C$ of some $C$ in $\underline{C}^{*}$ with countable domain and compact values.

A partial isomorphism between objects $a$ and $b$ of $\underline{C}$ is a pair of arrows $a \leftarrow d \rightarrow b$ in $\underline{C}$ (recall that arrows of $\underline{C}$ are monics). Two objects $a$ and $b$ are partially isomorphic, written $a \cong_{p} b$, iff there is a nonempty set $I$ of partial isomorphisms between $a$ and $b$ such that for every $a \leftarrow d \rightarrow b$ in $I$, every compact arrow to $a$ or $b$ factors through an extension in $I$ of $a \leftarrow d \rightarrow b$, i.e.,

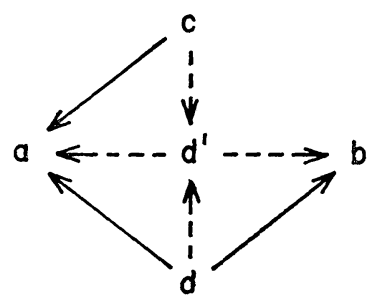

is completable with $a \leftarrow d^{\prime} \rightarrow b$ in $I$. If $I$ is as above, we write $I: a \cong_{p} b$. This definition is due to Karp [6]; see [1] for details. Two objects are partially isomorphic iff player II has a winning strategy in an appropriate Ehrenfeucht-Fraïssé game.

For any functor $\varphi: \underline{C} \times \cdots \times \underline{C} \rightarrow \underline{C}$ and arrows $a_{1} \rightarrow b_{1}, \cdots, a_{n} \rightarrow$ $b_{n}$, let $\varphi\left(a_{1}, \cdots, a_{n}\right) \rightarrow \varphi\left(b_{1}, \cdots, b_{n}\right)$ be the arrow $\varphi\left(a_{1} \rightarrow b_{1}, \cdots, a_{n} \rightarrow b_{n}\right)$. A functor $\varphi: \underline{C} \times \cdots \times \underline{C} \rightarrow \underline{C}$ is a constructor iff for any objects $a_{1}$, $\cdots, a_{n} \in \underline{C}$, every compact arrow $c \rightarrow \varphi\left(a_{1}, \cdots, a_{n}\right)$ factors through $\varphi\left(c_{1}, \cdots, c_{n}\right) \rightarrow \varphi\left(a_{1}, \cdots, a_{n}\right)$ for some compact arrows $c_{1} \rightarrow a_{1}, \cdots, c_{n} \rightarrow$ $a_{n}$. Zero-ary functors $1 \rightarrow \underline{C}$ are also regarded as constructors. Evidently the identity functor on $\underline{C}$ is a constructor and a composition of constructors is a constructor. For any set $\Phi$ of constructors let $\bar{\Phi}$ be the smallest set including $\Phi$ and the identity functor on $\underline{C}$ and closed under composition.

It is not hard to verify that if every object of $\underline{C}$ is a directed colimit of compacts, then $\varphi$ is a constructor iff $\varphi$ preserves directed colimits. 
Suppose each component of $\underline{C}$ has an initial object. Let $\Phi$ be a set of constructors on $\underline{C}$. For any $\varphi \in \Phi$, any objects $a_{1}, \cdots, a_{n} \in \underline{C}$ whose components have initial objects $p_{1}, \cdots, p_{n}$ respectively, let $\varphi\left(p_{1}\right.$, $\left.\cdots, p_{n}\right) \rightarrow \varphi\left(a_{1}, \cdots, a_{n}\right)$ be $\varphi\left(p_{1} \rightarrow a_{1}, \cdots, p_{n} \rightarrow a_{n}\right)$ where $p_{i} \rightarrow a_{i}$ is the unique arrow from $p_{i}$ to $a_{i}$. A symmetric binary relation $R$ on the objects of $\underline{C}$ is $\Phi$-decomposable iff for any objects $a$ and $b$ in $\underline{C}$ (1) $a R b$ implies $a$ and $b$ are in the same component of $\underline{C}$ and (2) $a R b$ and $c \rightarrow a$ compact implies there is a $\varphi$ in $\bar{\Phi}, a_{1}, \cdots, a_{n}, b_{1}, \cdots, b_{n}$ in $\underline{C}$, and isomorphisms $a \cong \varphi\left(a_{1}, \cdots, a_{n}\right)$ and $\varphi\left(b_{1}, \cdots, b_{n}\right) \cong b$ such that $a_{1} R b_{1}, \cdots, a_{n} R b_{n}$, and $c \rightarrow a$ factors through $\varphi\left(p_{1}, \cdots, p_{n}\right) \rightarrow \varphi\left(a_{1}, \cdots\right.$, $\left.a_{n}\right) \cong a$ where $p_{i}$ is an initial object of the common component of $a_{i}$ and $b_{i}$. (See the last section for examples.)

The back-and-forth theorems. The following lemma is related to the final functor theorem [7, p. 213].

LeMma 1. For any compactly generated category_C of monics,

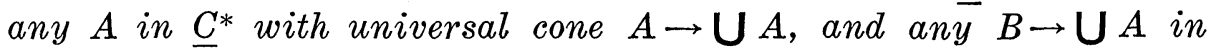
$\underline{C}^{*}$, if every component of $A \rightarrow \bigcup A$ factors through a component of $B \rightarrow \bigcup A$, then $\bigcup A$ is a colimit of $B$.

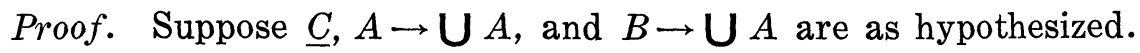
We claim that $B \rightarrow \mathrm{U} A$ is a universal cone. Given $B \rightarrow d$, let $A \rightarrow d$ be the unique arrow such that for any $A \rightarrow \bigcup A$ component $a \rightarrow \bigcup A$, the corresponding $A \rightarrow d$ component is $a \rightarrow d=a \rightarrow b \rightarrow d$ where (*) $b \rightarrow d$ is a $B \rightarrow d$ component and $a \rightarrow b$ is an arrow such that $a \rightarrow b \rightarrow$ $\mathrm{U} A=a \rightarrow \mathrm{U} A$ where $b \rightarrow \mathrm{U} A$ is the $B \rightarrow \mathrm{U} A$ component corresponding to $b \rightarrow d$. To show that $a \rightarrow d$ is independent of the choice of $b$, let $a \rightarrow b_{1} \rightarrow d$ and $a \rightarrow b_{2} \rightarrow d$ satisfy $(*)$. Thus, in the diagram below with $c \rightarrow a, c \rightarrow b_{1}$, and $\bigcup A \rightarrow d$ omitted, the triangles $H$ commute by hypothesis. Since $B$ has a directed domain, there are arrows $b_{1} \rightarrow b_{3} \leftarrow b_{2}$ and components $b_{3} \rightarrow \mathbf{U} A$ and $b_{3} \rightarrow d$ such that the triangles $N$ commute by naturality.

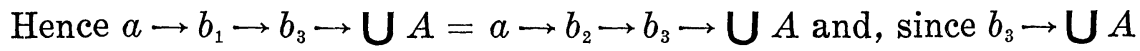
is monic, $a \rightarrow b_{1} \rightarrow b_{3}=a \rightarrow b_{2} \rightarrow b_{3}$. Hence $a \rightarrow b_{1} \rightarrow d=a \rightarrow b_{2} \rightarrow d$. Finally, these components "add up" to an arrow (cone) $A \rightarrow d$ since if $A$ assigns $a \rightarrow a^{\prime}$ to an arrow of its domain, if $a \rightarrow \bigcup A$ and $a^{\prime} \rightarrow$ $\bigcup A$ are the associated $A \rightarrow \bigcup A$ components and if $a \rightarrow d=a \rightarrow b \rightarrow d$ and $a^{\prime} \rightarrow d=a^{\prime} \rightarrow b^{\prime} \rightarrow d$ are the corresponding arrows satisfying $\left(^{*}\right)$; then, letting $a \rightarrow b^{\prime}=a \rightarrow a^{\prime} \rightarrow b^{\prime}$ and noting that $a \rightarrow b^{\prime} \rightarrow d$ satisfies $\left(^{*}\right)$, we have $a \rightarrow a^{\prime} \rightarrow d=a \rightarrow a^{\prime} \rightarrow b^{\prime} \rightarrow d=a \rightarrow b^{\prime} \rightarrow d=a \rightarrow d$, the last equality due to the independence of $a \rightarrow d$ from the choice of $b$. By universality of $A \rightarrow \bigcup \begin{aligned} & \mathrm{U} \\ & \text {, there is a } \bigcup\end{aligned} A \rightarrow d$ such that $A \rightarrow$ 


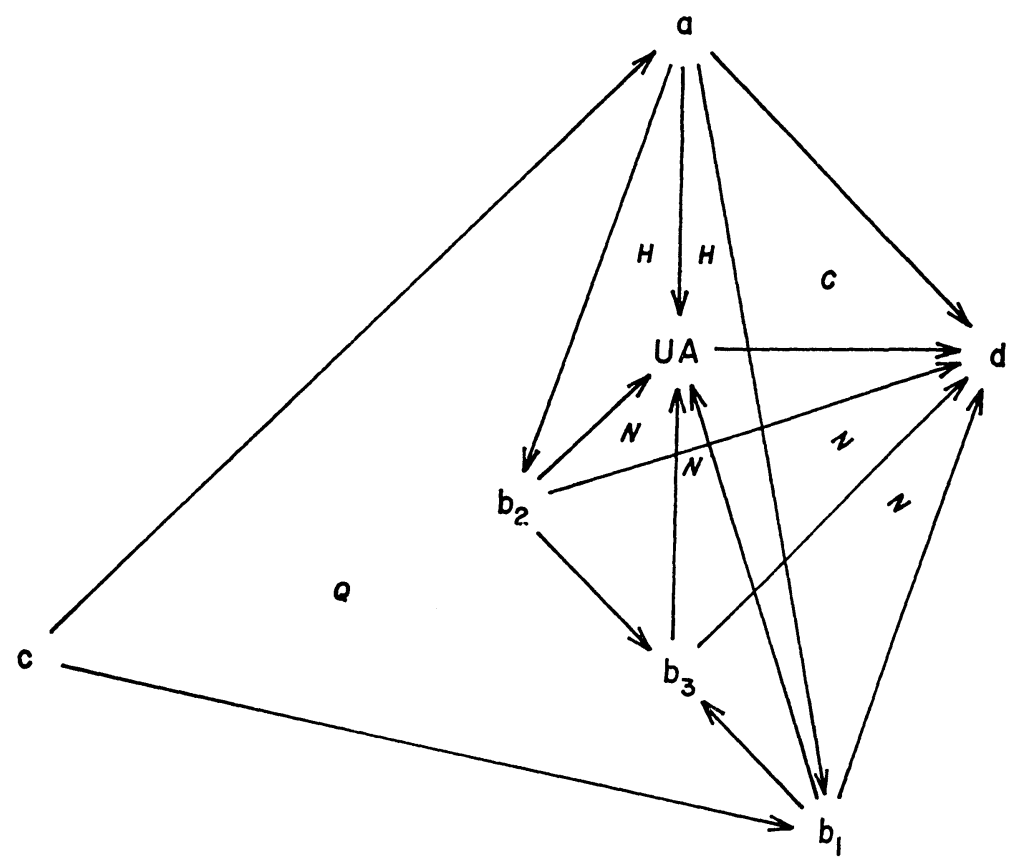

$\mathrm{U} A \rightarrow d=A \rightarrow d$. We show that $B \rightarrow \cup A \rightarrow d=B \rightarrow d$. Since $\underline{C}$ is compactly generated, it suffices to show that $c \rightarrow b \rightarrow \bigcup \begin{aligned} & \mathrm{U} A \rightarrow d=c \rightarrow \\ & \mathrm{c}\end{aligned}$ $b \rightarrow d$ for every pair $b \rightarrow \bigcup A$ and $b \rightarrow d$ of corresponding components of $B \rightarrow \bigcup A$ and $B \rightarrow d$ and every compact arrow $c \rightarrow b$. Suppose $b_{1} \rightarrow \bigcup A$ and $b_{1} \rightarrow d$ are corresponding components of $B \rightarrow \bigcup A$ and

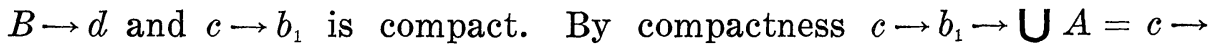
$a \rightarrow \bigcup A$ for some $A \rightarrow \bigcup A$ component $a \rightarrow \bigcup A$. Let $a \rightarrow d$ be the $A \rightarrow d$ component corresponding to $a \rightarrow \bigcup A$. Then by definition of $A \rightarrow d, a \rightarrow d=a \rightarrow b_{2} \rightarrow d$ for some $B \rightarrow d$ component $b_{2} \rightarrow d$ and some

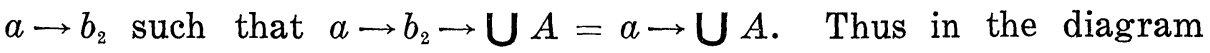
above, this time with $a \rightarrow b_{1}$ omitted, the triangle $H$ commutes by hypothesis and the triangle $C$ by choice of $\cup A \rightarrow d$. Since $B$ has a directed domain, there are arrows $b_{1} \rightarrow b_{3} \leftarrow b_{2}$ and components $b_{i} \rightarrow \cup A$ and $b_{i} \rightarrow d$ such that the triangles $N$ commute by naturality. Now $c \rightarrow$

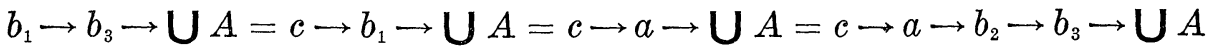
and $b_{3} \rightarrow \bigcup A$ is monic so $c \rightarrow b_{1} \rightarrow b_{3}=c \rightarrow a \rightarrow b_{2} \rightarrow b_{3}$ and the quintilateral $Q$ commutes. Using the commutativity of the triangles $H$, $N, C$, and $a b_{2} d$ and the quadrilateral $c b_{1} \cup A a, c \rightarrow b_{1} \rightarrow \bigcup A \rightarrow d=c \rightarrow$ $a \rightarrow \bigcup A \rightarrow d=c \rightarrow a \rightarrow d=c \rightarrow a \rightarrow b_{2} \rightarrow d=c \rightarrow a \rightarrow b_{1} \rightarrow b_{3} \rightarrow d=$ $c \rightarrow b_{1} \rightarrow b_{3} \rightarrow d=c \rightarrow b_{1} \rightarrow d$. Hence $B \rightarrow \bigcup A \rightarrow d=B \rightarrow d$.

To see that $\bigcup A \rightarrow d$ is the unique such arrow, use the same diagram, this time with $c, b_{1}$, and $b_{3}$ omitted and $\bigcup A \rightarrow d$ replaced by $\mathrm{U} A \rightarrow^{\prime} d$, to show that if $B \rightarrow \mathrm{U} A \rightarrow^{\prime} d=B \rightarrow d$, then $A \rightarrow \mathbf{U} A \rightarrow^{\prime} d=$

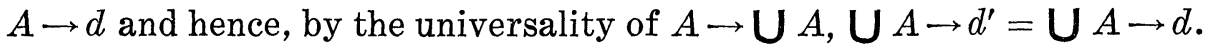


THEOREM 2. (Countable and $\cong{ }_{p}$ implies $\cong$.) If $a$ and $b$ are countable objects in a compactly generated category of monics, then $a \cong_{p} b$ implies $a \cong b$.

Proof. Let $A \rightarrow a$ and $B \rightarrow b$ be universal cones from functors with countable domains and compact values; let $a_{1} \rightarrow a, a_{3} \rightarrow a, a_{5} \rightarrow$ $a, \cdots$ and $b_{2} \rightarrow b, b_{4} \rightarrow b, \cdots$ be enumerations of their (compact) components; and let $I: a \cong_{p} b$. Let $a \leftarrow d_{0} \rightarrow b$ be any element of $I$. Given arrows $a \leftarrow d_{k} \rightarrow b$ in $I$ and $d_{k-1} \rightarrow d_{k}, k=1, \cdots, i-1$, such that the solid part of the diagram below commutes, let $a \leftarrow d_{i} \rightarrow b$ in $I$ be an extension of $a \leftarrow d_{i-1} \rightarrow b$ via some $d_{i-1} \rightarrow d_{\imath}$ such that $a_{\imath} \rightarrow a$, if $i$ is odd, or $b_{\imath} \rightarrow b$, if $i$ is even, factors through $a \leftarrow d_{i} \rightarrow b$.

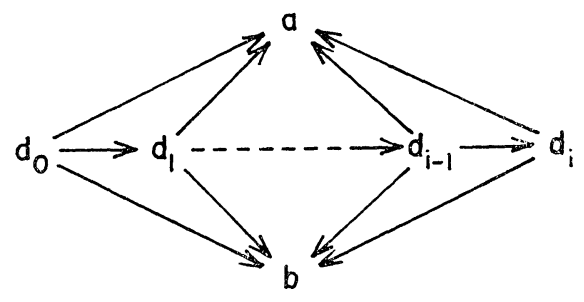

Let $D$ in $\underline{C}^{*}$ be the functor from the directed category $\omega$ for which $D(i)=d_{i}$ and $D(i \rightarrow i+1)=d_{i} \rightarrow d_{\imath+1}$, i.e., $D=" d_{0} \rightarrow d_{1} \rightarrow$ $d_{2} \cdots "$, and let $D \rightarrow a$ be the arrow of $C^{*}$ whose $i$ th component is $d_{i} \rightarrow a$. By construction, every component of $A \rightarrow a$ factors through a component of $D \rightarrow a$ and so, by Lemma $1, a$ is a colimit of $D$. Likewise $b$ is a colimit of $D$ and hence $a \cong b$.

THeOREM 3. (Decomposably related implies $\cong_{p}$.) If every component of the category $\underline{C}$ has an initial object, if $\Phi$ is a set of constructors on $\underline{C}$, and $i \bar{f} R$ is a $\Phi$-decomposable relation, then aRb implies $a \cong_{p} b$.

Proof. Suppose $\Phi$ and $R$ are as hypothesized and suppose $a R b$. Let $I$ be the set of partial isomorphisms from $a$ to $b$ of the form $a \cong \varphi\left(a_{1}, \cdots, a_{n}\right) \leftarrow \varphi\left(p_{1}, \cdots, p_{n}\right) \rightarrow \varphi\left(b_{1}, \cdots, b_{n}\right) \cong b$, where $\varphi$ is in $\bar{\Phi}$, $a_{i} R b_{\imath}, p_{\imath}$ is an initial object of the common component of $a_{i}$ and $b_{i}$, $a \cong \varphi\left(a_{1}, \cdots, a_{n}\right)$ and $\varphi\left(b_{1}, \cdots, b_{n}\right) \cong b$ are isomorphisms, and for $d_{i}$ in the component of $p_{i}$ and $p_{i} \rightarrow d_{2}$ the unique arrows, $\varphi\left(p_{1}, \cdots, p_{n}\right) \rightarrow$ $\varphi\left(d_{1}, \cdots, d_{n}\right)$ is $\varphi\left(p_{1} \rightarrow d_{1}, \cdots, p_{n} \rightarrow d_{n}\right)$.

If $p$ is an initial object of the common component of $a$ and $b$, then, since the identity is in $\bar{\Phi}, a \leftarrow p \rightarrow b$ is in $I$ and hence $I$ is nonempty. If $a \cong \varphi\left(a_{1}, \cdots, a_{n}\right) \leftarrow \varphi\left(p_{1}, \cdots, p_{n}\right) \rightarrow \varphi\left(b_{1}, \cdots, b_{n}\right) \cong b$ is in $I$ and $c \rightarrow a$ compact, then, since $\phi$ is a constructor, the compact arrow $c \rightarrow \varphi\left(\alpha_{1}, \cdots, a_{n}\right)=c \rightarrow a \cong \varphi\left(a_{1}, \cdots, \alpha_{n}\right)$ factors through $\varphi\left(c_{1}\right.$, 
$\left.\cdots, c_{n}\right) \rightarrow \varphi\left(a_{1}, \cdots, a_{n}\right)$ for some compacts $c_{i} \rightarrow a_{i}$. Since $R$ is $\Phi$ decomposable, each $c_{i} \rightarrow a_{i}$ factors through $\varphi\left(p_{i 1}, \cdots, p_{i_{p_{i}}}\right) \rightarrow \varphi_{i}\left(a_{i 1}\right.$, $\left.\cdots, a_{i n_{i}}\right) \cong a_{i}$ for some $\varphi_{i}$ in $\bar{\Phi}$, some $a_{i j}$ and $b_{i j}$ with $a_{i j} R b_{i j}$ and some $a_{i} \cong \varphi_{i}\left(a_{i 1}, \cdots, a_{i n_{i}}\right)$ and $\varphi_{i}\left(b_{i 1}, \cdots, b_{i n_{i}}\right) \cong b_{i}$. Hence $c \rightarrow a$ factors through $a \cong \varphi\left(a_{1}, \cdots, a_{n}\right) \cong \varphi\left(\varphi_{1}\left(a_{11}, \cdots, a_{1 n_{1}}\right), \cdots, \varphi_{n}\left(a_{n 1}, \cdots, a_{n n_{n}}\right)\right) \leftarrow$ $\varphi\left(\varphi_{1}\left(p_{11}, \cdots, p_{1 n_{1}}\right), \cdots, \varphi_{n}\left(p_{n 1}, \cdots, p_{n n_{n}}\right)\right) \rightarrow \varphi\left(\varphi_{1}\left(b_{11}, \cdots, b_{1 n_{1}}\right), \cdots, \varphi_{n}\left(b_{n 1}\right.\right.$, $\left.\left.\cdots, b_{n n_{n}}\right)\right) \cong \varphi\left(b_{1}, \cdots, b_{n}\right) \cong b$ which is in $I$ and which extends $a \cong$ $\varphi\left(a_{1}, \cdots, a_{n}\right) \leftarrow \varphi\left(p_{1}, \cdots, p_{n}\right) \rightarrow \varphi\left(b_{1}, \cdots, b_{n}\right) \cong b$ via $\varphi\left(p_{1}, \cdots, p_{n}\right) \rightarrow$ $\varphi\left(\varphi_{1}\left(p_{11}, \cdots, p_{1 n_{1}}\right), \cdots, \varphi_{n}\left(p_{n 1}, \cdots, p_{n n_{n}}\right)\right)$. Hence $I: a \cong_{p} b$.

Suppose for the remainder of the section that $\underline{C}$ has an initial object $p$. Then condition (1) of the $\Phi$-decomposability definition is trivial.

Definition. With respect to a given set $\Phi$ of constructors on $\underline{C}$, an object $b$ is a factor of an object $a$ iff $a \cong \varphi\left(a_{1}, \cdots, a_{n}, b\right)$ for some $a_{1}, \cdots, a_{n}$ and some $\varphi \in \bar{\Phi} . \quad \underline{C}$ is $\Phi$-decomposable iff every compact arrow $c \rightarrow a$ factors through $\varphi(p, \cdots, p) \rightarrow \varphi\left(a_{1}, \cdots, a_{n}\right) \cong a$ for some $a_{1}, \cdots, a_{n}$, some $\varphi \in \bar{\Phi}$, and some $\varphi\left(a_{1}, \cdots, a_{n}\right) \cong a$.

Note. $\underline{C}$ is $\Phi$-decomposable iff the equality relation on $\underline{C}$ is $\Phi$ decomposable.

Definition. For any set $\Phi$ of constructors and any object $a$, the $\Phi$-factor algebra of $a$ is the partial algebra consisting of isomorphism types of factors of $a$ under the operations of $\Phi$ plus a constant for the isomorphism type of $a$. (See the next section for examples.)

CoRollary 4. If $\underline{C}$ is $\Phi$-decomposable and objects a and $b$ have isomorphic factor algebras, then $a \cong_{p} b$.

Proof. Let $R$ be the relation on $\underline{C}$ such that $a R b$ iff $a$ and $b$ have isomorphic $\Phi$-factor algebras. Suppose $a R b$ and $c \rightarrow a$ is a compact arrow. Then by decomposability of $\underline{C}, c \rightarrow a$ factors through $\varphi(p, \cdots, p) \rightarrow \varphi\left(a_{1}, \cdots, a_{n}\right) \cong a$ for some $\varphi \in \bar{\Phi}$ and some $\varphi\left(a_{1}, \cdots\right.$, $\left.a_{n}\right) \cong a$. Let $f$ be an isomorphism from the $\Phi$-factor algebra of $a$ to that of $b$, let $\bar{a}_{i}$ be the isomorphism type of $a_{i}$, and let $b_{i}$ be of the type $f\left(\bar{a}_{i}\right)$. Then $b \cong \varphi\left(b_{1}, \cdots, b_{n}\right)$ and $a_{i} R b_{i}$. Hence $R$ is $\Phi$ decomposable and, by Theorem 3 , $a R b$ implies $a \cong_{p} b$.

EXAMPLES. In the following we give examples of constructors, decomposable relations, and factor algebras in the categories of sets, linear orders, Boolean algebras, bordered surfaces, and structures of a first order language. 
Sets. Let Set be the category of sets and $1-1$ functions. The empty set is the initial object and the finite and countable sets are the compact and countable objects respectively. Let $\varphi:$ Set $\rightarrow$ Set be the functor such that $\varphi(X)=X \cup\{X\}$ for any set $X$ and $\varphi(f)=$ $f \cup\{\langle$ domain $f$, range $f\rangle\}$ for any function $f$. Then $\varphi$ is a constructor. If $R$ is a relation on sets such that $X R Y$ iff $X$ and $Y$ have the same finite cardinality or are both infinite, then $R$ is $\{\varphi\}$-decomposable. The $\{\varphi\}$-factor algebra of the set $\{0,1, \cdots, n-1\}$ is $\langle\{0,1$, $\cdots, n\}, S, n\rangle$ where $S(i)$ is $i+1$ if $i<n$ and undefined if $i=n$. The $\{\varphi\}$-factor algebra of $\omega=\{0,1, \cdots\}$ is (isomorphic to) $\langle\omega \cup\{\infty\}$, $S, \infty\rangle$ where $S(\alpha)=\alpha+1$ if $\alpha \in \omega$ and $\infty$ if $\alpha=\infty$.

Linear orders. Let Lin be the category of linear orders and 1-1 order preserving functions. The empty order is the initial object and the finite and countable orders are the compact and countable objects respectively. Let $\varphi:$ Lin $\times$ Lin $\rightarrow$ Lin be a functor such that $\varphi(K, L)$ is an order consisting of an initial segment of type $K$ followed by a new point followed by a final segment of type $L$ and for functions $f$ and $g, \varphi(f, g)$ is, essentially, $f$ on the first segment, the new point goes to the new point, and $g$ on the final segment. Then $\phi$ is a constructor. If $R$ is the relation such that $K R L$ iff $K$ and $L$ are both dense linear orders without end points, then $R$ is $\{\varphi\}$ decomposable. If $R$ is any symmetric relation on linear orders such that (1) $K R L$ implies $K$ is empty iff $L$ is and (2) $K R L$ and $a \in k$ implies there is a $b \in L$ such that $\left\langle\left\{x \in K: x<_{K} a\right\},<_{K}\right\rangle R\left\langle\left\{x \in L: x<_{L} b\right\}\right.$, $\left.<_{L}\right\rangle$ and $\left\langle\left\{x \in K: x>_{K} a\right\},<_{K}\right\rangle R\left\langle\left\{x \in L: x>_{L} b\right\},<_{L}\right\rangle$, then $R$ is $\{\varphi\}-$ decomposable. The $\{\varphi\}$-factor algebra of the empty order is $\langle\{1\}$, + , 1) where $1+1$ is undefined. The $\{\varphi\}$-factor algebra of the rationals with the usual order is $\langle\{\infty\},+, \infty\rangle$ where $\infty+\infty=\infty$.

Boolean algebras. Let Bool be the category of Boolean algebras with two or more elements and monomorphisms. The two-element Boolean algebra is the initial object and the finite and countable algebras are the compact and countable objects respectively. Let $\varphi:$ Bool $\times$ Bool $\rightarrow$ Bool be the cartesian product functor. Then $\phi$ is a constructor. If $R$ is the relation such that $A R B$ iff $A$ and $B$ are atomless Boolean algebras, then $R$ is $\{\varphi\}$-decomposable. If $R$ is any symmetric relation on Boolean algebras such that (1) $A R B$ implies $A$ is the two-element algebra iff $B$ is and (2) $A R B$ and $a \in A$ implies there is a $b \in B$ such that $A[a] R B[b]$ and $A[-a] R B[-b]$ where $A[a]$ is the ideal (considered as a Boolean algebra) generated by $a$, then $R$ is $\{\varphi\}$-decomposable. Relations of this type were first studied by Vaught [13] who showed that any two countable Boolean algebras related by such a relation were isomorphic. Decomposability is 
simply a categorical generalization of conditions (1) and (2). The $\{\varnothing\}$ factor algebras of the two-element algebra and the atomless algebra are the partial algebras $\langle\{1\},+, 1\rangle$ and $\langle\{\infty\},+, \infty\rangle$ respectively defined in the previous example. For primitive Boolean algebras, $\{\varnothing\}$-factor algebras are nothing more than algebraic versions of the structure diagrams of Hanf [3] and Pierce [10] which have played an important role in classifying such Boolean algebras and determining isomorphism types of Lindenbaum-Tarski algebras $[3,9,12]$.

Bordered surfaces. Let Sur be the category whose objects are bordered orientable surfaces (orientable two-dimensional manifolds whose boundary is a disjoint union of simple closed curves) each with a designated homeomorphism from the closed unit disc onto a subspace of the surface's interior and whose arrows are homeomorphisms from one surface onto a subsurface of a second such that the designated map of the first composed with the arrow is the designated map of the second and such that a boundary curve of the first is either carried onto a boundary curve of the second or into the interior of the second. The closed disc with the identity map as the designated map is the initial object, the compact bordered surfaces are the compact objects (this is false if the boundary curve condition on arrows is dropped), and the separable bordered surfaces are the countable objects. Let $C$ be a cylinder - finite length, closed, and open at both ends - with a designated (map from the) disc in its interior. Let $\varphi$ : Sur $\times$ Sur $\rightarrow$ Sur be a functor such that for any objects $S$ and $T, \varphi(S, T)$ is a surface obtained by cutting out the interiors of the designated disc of $S$ and $T$ and gluing their perimeters to the open ends of $C$ ( $C$ becomes a tube joining the two surfaces) and letting the designated disc of $C$ be the designated disc of the result; and such that for any arrows $f$ and $g, \varphi(f, g)$ is, essentially, $f$ on the first surface, $g$ on the second, and the identity on $C$. Let $\sigma$ and $\tau$ be the 0 -ary functors whose unique values are the sphere and torus respectively with designated discs. Then $\varphi, \sigma$, and $\tau$ are constructors. If $R$ is the relation between surfaces such that $S R T$ iff $S$ and $T$ have the same number of boundary components, the same genus, and homeomorphic ideal boundaries (see [11]), then $R$ is $\{\varphi, \sigma, \tau\}$-decomposable. The $\{\varphi, \sigma, \tau\}$ factor algebra of the surface of a solid semi-infinite $\operatorname{rod}$ is $\langle\{s, t, 1\},+$, $s, t, 1\rangle$ where $s+s=s, s+t=t+s=t, s+1=1+s=1$, and all other sums are undefined. The $\{\varphi, \sigma, \tau\}$-factor algebra of the surface of an infinite binary tree with solid branches of nonzero width is $\langle\{s, t, \infty\},+, s, t, \infty\rangle$ where $s+s=s, s+t=t+s=t, s+\infty=\infty+s=$ $\infty, \infty+\infty=\infty$, and all other sums are undefined.

Structures. Given a first-order language with at least one constant 
symbol, let Str be the category whose objects are finite expansions of structures of the language via constants, i.e., structures of the form ( $\mathfrak{l}, a_{1}, \cdots, a_{n}$ ) where $\mathfrak{i}$ is a structure of the language and $a_{1}, \cdots$, $a_{n} \in \mathfrak{R}$, and whose arrows are isomorphisms from one structure to a substructure of a second, i.e., monomorphisms which preserve quantifier-free formulas. For any object $\mathfrak{B}$ of $\mathbf{S t r}$ the initial object of its component is Prime ( $\mathfrak{B})$, the substructure of $\mathfrak{B}$ generated by its constants. Finitely generated structures and countably generated structures are the compact and countable objects respectively. Let $\varphi:$ Str $\rightarrow$ Str be the functor such that for any structure 2 of the language $\varphi(\mathfrak{N})=\mathfrak{X}$ and for any $a_{1}, \cdots, a_{n} \in \mathfrak{N}, \varphi\left(\left(\mathfrak{N}, a_{1}, \cdots, a_{n}\right)\right)=(\mathfrak{N}$, $\left.a_{1}, \cdots, a_{n-1}\right)$. Then $\varphi$ is a constructor. If $R$ is the relation such that $\mathfrak{Z} R \mathfrak{B}$ iff $\mathfrak{R}$ and $\mathfrak{B}$ are elementarily equivalent $\omega$-saturated structures, then $R$ is $\{\varphi\}$-decomposable. If $R$ is a symmetric relation such that (1) $\mathfrak{Z} R \mathfrak{B}$ implies Prime $\mathfrak{Z} \cong$ Prime $\mathfrak{B}$ and (2) $\mathfrak{R} R \mathfrak{B}$ and $a \in \mathfrak{N}$ implies there is a $b \in \mathfrak{B}$ such that $(\mathfrak{R}, a) R(\mathfrak{B}, b)$, then $R$ is $\{\varnothing\}$-decomposable.

\section{REFERENCES}

1. Jon Barwise, Back and forth through infinitary logic, Studies in Model Theory (M. D. Morley, Editor), The Mathematical Association of America, 1973.

2. Georg Cantor, Beiträge zur Begründung der iransfiniten Mergenlehre, Math. Ann., 96 (1895), 481-512.

3. William Hanf, Primitive Boolean algebras, Proceedings of the Symposium in Honor of Alfred Tarski, (Berkeley, 1971), Amer. Math. Soc., Providence, R. I., 25 (1974), 75-90.

4. Henkin, Monk, and Tarski, Cylindric Algebras, North-Holland Publishing Company, Amsterdam, 1971.

5. Irving Kaplansky, Infinite Abelian Groups, Michigan Press, Ann Arbor, 1969.

6. Carol Karp, Finite Quantifier Equivalence, The Theory of Models, North-Holland Publishing Company, Amsterdam, 1965.

7. Saunders Mac Lane, Categories for the Worling Mathematician, Springer-Verlag, New York, 1971.

8. M. Morley and R. L. Vaught, Homogeneous universal models, Math. Scand., 11 (1962), 37-57.

9. Dale Myers, The Boolean algebras of abelian groups and vell-orders, J. Symbolic Logic, 39 (1974), 452-458.

10. R. S. Pierce, Compact zero-dimensional metric spaces of finite type, Memoirs of the Amer. Math Soc., no. 130 (1972).

11. Ian Richards, On the classification of noncompact surfaces. Trans. Amer. Math. Soc., 106 (1963), 259-269.

12. Roger Simons, The Boolean algebra of sentences of the theory of a function, Ph. D. thesis, Berkeley, 1972.

13. R. L. Vaught, Topics in the theory of arithmetical classes and Boolean algebras, Ph. D. thesis, Berkeley, 1954.

Received June 11, 1974.

UNIVERSITY OF HAWAII 



\section{PACIFIC JOURNAL OF MATHEMATICS}

\section{EDITORS}

RICHARD ARENS (Managing Editor)

University of California

Los Angeles, California 90024

\section{R. A. Beaumont \\ University of Washington \\ Seattle, Washington 98105}

\section{J. DugundJI}

Department of Mathematics

University of Southern California

Los Angeles, California 90007

D. Gilbarg and J. Milgram

Stanford University

Stanford, California 94305

\section{ASSOCIATE EDITORS}
E. F. BECKENBACH
B. H. NeumanN
F. WOLF
K. YOSHIDA

\section{SUPPORTING INSTITUTIONS}

UNIVERSITY OF BRITISH COLUMBIA
CALIFORNIA INSTITUTE OF TECHNOLOGY
UNIVERSITY OF CALIFORNIA
MONTANA STATE UNIVERSITY
UNIVERSITY OF NEVADA
NEW MEXICO STATE UNIVERSITY
OREGON STATE UNIVERSITY
UNIVERSITY OF OREGON
OSAKA UNIVERSITY

UNIVERSITY OF BRITISH COLUMBIA

UNIVERSITY OF CALIFORNIA

MONTANA STATE UNIVERSITY

NEW MEXICO STATE UNIVERSITY

OREGON STATE UNIVERSITY

OSAKA UNIVERSITY

\author{
UNIVERSITY OF SOUTHERN CALIFORNIA \\ STANFORD UNIVERSITY \\ UNIVERSITY OF TOKYO \\ UNIVERSITY OF UTAH \\ WASHINGTON STATE UNIVERSITY \\ UNIVERSITY OF WASHINGTON \\ AMERICAN MATHEMATICAL SOCIETY \\ NAVAL WEAPONS CENTER
}

The Supporting Institutions listed above contribute to the cost of publication of this Journal, but they are not owners or publishers and have no responsibility for its content or policies.

Mathematical papers intended for publication in the Pacific Journal of Mathematics should be in typed form or offset-reproduced, (not dittoed), double spaced with large margins. Underline Greek letters in red, German in green, and script in blue. The first paragraph or two must be capable of being used separately as a synopsis of the entire paper. Items of the bibliography should not be cited there unless absolutely necessary, in which case they must be identified by author and Journal, rather than by item number. Manuscripts, in triplicate, may be sent to any one of the editors. Please classify according to the scheme of Math. Reviews, Index to Vol. 39. All other communications should be addressed to the managing editor, or Elaine Barth, University of California, Los Angeles, California, 90024.

The Pacific Journal of Mathematics expects the author's institution to pay page charges, and reserves the right to delay publication for nonpayment of charges in case of financial emergency.

100 reprints are provided free for each article, only if page charges have been substantially paid. Additional copies may be obtained at cost in multiples of 50 .

The Pacific Journal of Mathematics is issued monthly as of January 1966. Regular subscription rate: $\$ 72.00$ a year (6 Vols., 12 issues). Special rate: $\$ 36.00$ a year to individual members of supporting institutions.

Subscriptions, orders for back numbers, and changes of address should be sent to Pacific Journal of Mathematics, 103 Highland Boulevard, Berkeley, California, 94708.

PUBLISHED BY PACIFIC JOURNAL OF MATHEMATICS, A NON-PROFIT CORPORATION

Printed at Kokusai Bunken Insatsusha (International Academic Printing Co., Ltd.), 270, 3-chome Totsuka-cho, Shinjuku-ku, Tokyo 160, Japan.

Copyright (C) 1973 by Pacific Journal of Mathematics Manufactured and first issued in Japan 


\section{Pacific Journal of Mathematics \\ Vol. 55, No. $2 \quad$ October, 1974}

Walter Allegretto, On the equivalence of two types of oscillation for elliptic

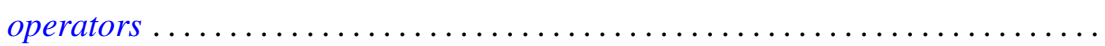

Edward Arthur Bertram, A density theorem on the number of conjugacy classes in

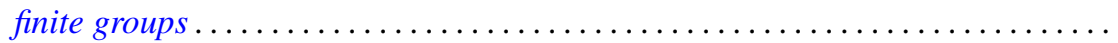

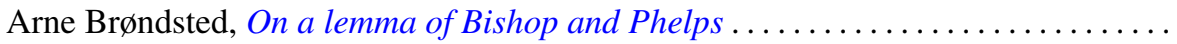

Jacob Burbea, Total positivity and reproducing kernels ..................

Ed Dubinsky, Linear Pincherle sequences . . . . . . . . . . . . . . . . . .

Benny Dan Evans, Cyclic amalgamations of residually finite groups .............

361

Barry J. Gardner and Patrick Noble Stewart, A "going down" theorem for certain

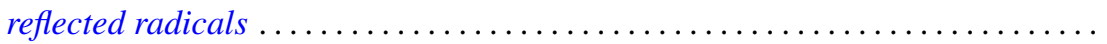

381

Jonathan Light Gross and Thomas William Tucker, Quotients of complete graphs:

revisiting the Heawood map-coloring problem ....................

Sav Roman Harasymiv, Groups of matrices acting on distribution spaces .........

Robert Winship Heath and David John Lutzer, Dugundji extension theorems for

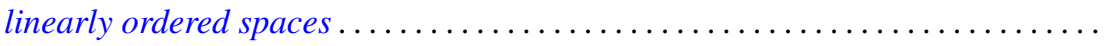

Chung-Wu Ho, Deforming p. l. homeomorphisms on a convex polygonal

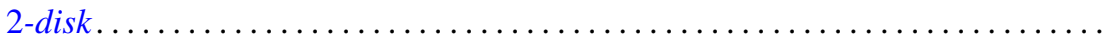

Richard Earl Hodel, Metrizability of topological spaces .................

Wilfried Imrich and Mark E. Watkins, On graphical regular representations of

cyclic extensions of groups .......................... 461

Jozef Krasinkiewicz, Remark on mappings not raising dimension of curves ..... . 479

Melven Robert Krom, Infinite games and special Baire space extensions . . . . . . 483

S. Leela, Stability of measure differential equations . . . . . . . . . . . . . . . . 489

M. H. Lim, Linear transformations on symmetric spaces . . . . . . . . . . . . . . . 499

Teng-Sun Liu, Arnoud C. M. van Rooij and Ju-Kwei Wang, On some group algebra modules related to Wiener's algebra $M_{1} \ldots \ldots \ldots \ldots \ldots \ldots \ldots \ldots \ldots \ldots \ldots$

Dale Wayne Myers, The back-and-forth isomorphism construction ............ 521

Donovan Harold Van Osdol, Extensions of sheaves of commutative algebras by

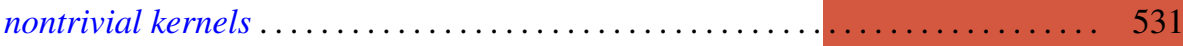

Alan Rahilly, Generalized Hall planes of even order ................... 543

Joylyn Newberry Reed, On completeness and semicompleteness of first countable

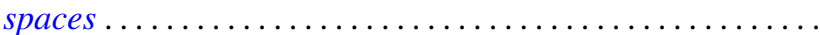

Alan Schwartz, Generalized convolutions and positive definite functions associated

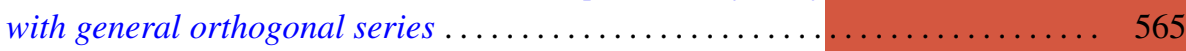

Thomas Jerome Scott, Monotonic permutations of chains . . . . . . . . . . . 583

Eivind Stensholt, An application of Steinberg's construction of twisted groups .... 595

Yasuji Takeuchi, On strongly radicial extensions . . . . ................. 619

William P. Ziemer, Some remarks on harmonic measure in space . . . . . . . . . . 629

John Grant, Corrections to: “Automorphisms definable by formulas” . . . . . . . . 639

Peter Michael Rosenthal, Corrections to: "On an inversion for the general

Mehler-Fock transform pair" ......................... 640

Carl Clifton Faith, Corrections to: "When are proper cyclics injective” . . . . . . 640 